
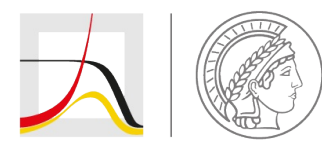

MAX PLANCK INSTITUTE

FOR DEMOGRAPHIC RESEARCH

Konrad-Zuse-Strasse 1 · D-18057 Rostock · Germany · Tel +49 (0) 3812081 - 0 · Fax +49 (0) 3812081 - $202 \cdot$ www.demogr.mpg.de

MPIDR Working Paper WP 2020-032 I September 2020

https://doi.org/10.4054/MPIDR-WP-2020-032

\title{
COVerAGE-DB: A database of age- structured COVID-19 cases and deaths
}

\author{
Tim Riffe I riffe@demogr.mpg.de \\ Enrique Acosta I acosta@demogr.mpg.de \\ COVerAGE-DB project team I coverage-db@demogr.mpg.de
}

This working paper has been approved for release by: Maarten J. Bijlsma (bijlsma@demogr.mpg.de),

Deputy Head of the Laboratory of Population Health.

(c) Copyright is held by the authors.

Working papers of the Max Planck Institute for Demographic Research receive only limited review. Views or opinions expressed in working papers are attributable to the authors and do not necessarily reflect those of the Institute. 


\title{
COVerAGE-DB: \\ A database of age-structured COVID-19 cases and deaths
}

Tim Riffe ${ }^{*}{ }^{1}$, Enrique Acosta $\dagger^{1}$, and COVerAGE-DB project team ${ }^{2}$

(*) corresponding

$(\dagger)$ co-first author

(1) Max Planck Institute for Demographic Research, Germany

$\left({ }^{2}\right)$ The full list of authors and contributions within the COVerAGE-DB project are presented at the end of the manuscript.

Suggested display authors:

Tim Riffe, Enrique Acosta, and the COVerAGE-DB team

\begin{abstract}
COVerAGE-DB is an open access database including cumulative counts of confirmed COVID-19 cases, deaths, and tests by age and sex. Original data and sources are provided alongside data and measures in age-harmonized formats. The database is still in development, and at this writing, it includes 87 countries, and 195 subnational areas. Cumulative counts of COVID-19 cases, deaths, and tests are recorded daily (when possible) since January 2020. Many time series thus fully capture the first pandemic wave and the beginning of later waves. An international team, composed of more than 60 researchers, contributed to the collection of data and metadata in COVerAGE-DB from governmental institutions, as well as to the design and implementation of the data processing and validation pipeline. We encourage researchers interested in supporting this project to send a message to the email:

coverage-db@demogr.mpg.de
\end{abstract}




\section{Data Resource Basics}

Information about pandemic dynamics is critical to understand the potential impacts on populations, design mitigation strategies, and evaluate the efficacy of their implementation. Centralization, standardization, and harmonization of data is critical to enable comparisons of the demographic impact of COVID-19 vis-à-vis differences in the age-compositions of confirmed infections and deaths. The international data landscape must keep pace with the global march of the pandemic, and researchers must work to triangulate the available data to create comparable measures to monitor and predict its demographic impacts.

The COVID Age Database (COVerAGE-DB) aims to provide global coverage of key demographic aspects of the COVID-19 pandemic as it unfolds in an up-to-date, transparent, and open-access format. COVerAGE-DB offers data with standardized count measures and harmonized age groups to allow comparisons between populations at national and subnational scales.

The database is currently under expansion through both the increase in coverage of national and subnational populations and the inclusion of more recent periods as the pandemic continues to unfold. At this writing, the database contains daily counts of COVID-19 cases, deaths, and tests performed by age and sex for $\mathbf{8 7}$ national and $\mathbf{1 9 5}$ subnational populations around the world, depending on the available data for each source. The date range available for each country or subpopulation varies. In several country series, the database includes the earliest confirmed cases in January, 2020. For most populations the database includes daily time series, beginning from an initial starting date when the data were first released or collected by our team. Fig. 1 displays a map of countries included in the database, indicating at least one subnational population from 12 countries. A detailed overview of data availability is given in a searchable table [external link] (https://bit.ly/3kVDrLD)

Figure 1. Availability of national- and subnational information on COVID-19 cases, deaths, and tests in the countries included in the database as of 24 August 2020. 


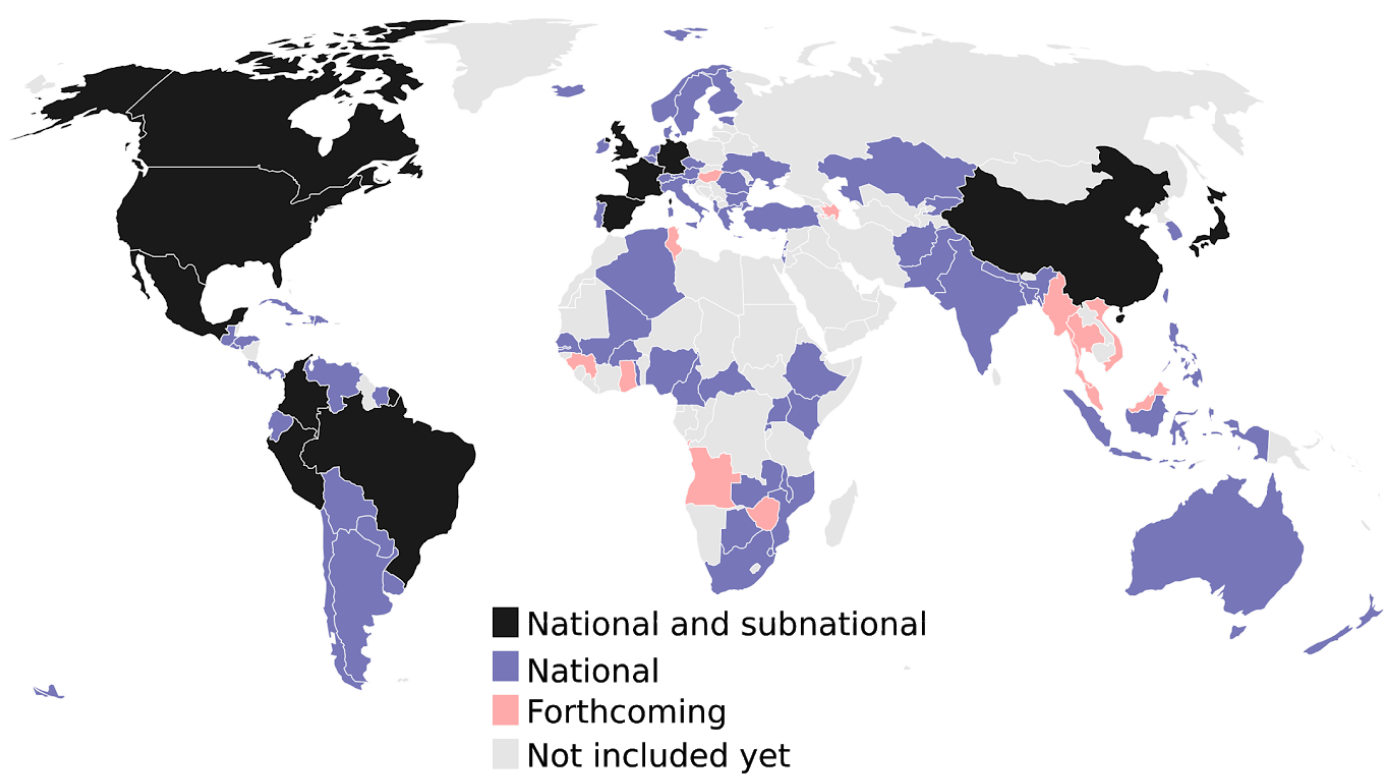

\section{Data Collected:}

Collection: Official counts of COVID-19 cases, deaths, and tests are extracted from reports published by official governmental institutions, such as health ministries and statistical offices. Depending on the source, data are collected in a variety of formats, including machine-readable files, pdf tables, html tables, interactive dashboards, press releases, official announcements via Twitter, and in a few instances, from digitized graphics. A full list of data sources is available in a dashboard view (https://bit.ly/20g1MxL).

Generally, COVID-19 cases, deaths and tests are reported as counts in 10-year age groups, but some sources report data in other metrics (fractions, percents, ratios) or as summary indicators such as case fatality ratios by age. Reported age intervals vary by source, ranging from single ages to 30-year or greater age bands, and sometimes reported age intervals change over time within sources. Usually data are reported as cross-sectional snapshots of cumulative counts, but some sources give full time series of new cases or deaths, in which case we cumulate counts over time. We also collect standard metadata on each of the sources to capture various characteristics of the collected data, such as the primary collection channels, definitions used, and notes on major disruptions or events. An overview of key fields from this metadata is shared as a spreadsheet (https://bit.ly/2FAmKFn). 
Production: All source data is entered into standard spreadsheet templates hosted in a central folder on Google Drive. Data entry into the templates is either manual or automatic depending on the source.

$\mathrm{R}$ programs collect data from the source templates and compile the merged input database. The merged input file is then subject to a series of automatic validity checks. Initial checks are carried out by the individual responsible for data collection and entry using an interactive application (https://mpidr.shinyapps.io/cleaning_tracker/). Data is then harmonized to standard metrics (counts), measures (cases, deaths, tests), and age bands (5and 10-year age intervals). Harmonization procedures include various kinds of rescaling to ensure coherence in marginal sums. Age group harmonization is done using the penalized composite link model, ${ }^{1}$ which was designed for splitting histograms of count data.

The complete details on all steps of production are available in the COVerAGE-DB Method Protocol, which is publicly available on the web (https://osf.io/jenw3/). A table listing which adjustments are applied to each population is available on the project website (https://bit.ly/2E61BSV). Both the merged input database and the harmonized output files are uploaded daily as zipped csv files to an Open Science Framework repository (OSF) (https://osf.io/mpwjq/). A GitHub repository (https://bit.ly/2YbtPCI), which is linked to OSF, contains all R scripts used in the complete production pipeline, including compilation, diagnostics, and harmonization.

\section{Data Resource Use:}

Since collection efforts began for COVerAGE-DB in late March 2020, we are aware of 6 studies using the data, many of which provide R code online and are fully reproducible. These studies aim to: (i) explore country differences in the age distribution of COVID-19 deaths, ${ }^{2}$ (ii) assess the contribution of infection case age-structure and age-specific fatality to between- and within-country differences in case fatality rates (CFR) associated with the COVID-19, ${ }^{3}$ (iii) produce a standard age-specific case fatality rate pattern for an indirect demographic method to estimate COVID-19 total infections ${ }^{4}$ (iv) analyze the association between intergenerational relationships and COVID-19 fatality rates, ${ }^{5}(\mathrm{v})$ estimate years of life lost due to Covid $-19,{ }^{6}$ and (vi) calculate pooled sex ratios of age-specific CFRs of COVID-19 in Europe. ${ }^{7}$ The database is also used to monitor COVID-19 impacts in particular age ranges, for instance, UNICEF uses the database for monitoring the burden of the pandemic on the infant, child, and juvenile mortality around the world.

As an example of the analyses that COVerAGE-DB enables, Fig. 2 displays changes in the relation between age-specific deaths and cases in Colombia, 
inspired by Fig. 1 of Dudel et al. ${ }^{3}$ We divide both cases and deaths in each age band by the respective population sizes. Diagonal lines indicate implied age-specific case fatality rates. The graphic illustrates a sharp increase in CFR over age for each sex, and it also displays considerable sex differences. For instance, men aged 60-69 in Colombia have almost the same case fatality rates (approximately $12 \%$ risk of death after COVID-19 infection is diagnosed) as women aged 70-79.

Figure 2. Relation between deaths and cases per 100,000 population by age group and sex in Colombia, until 22 August 2020. Diagonal lines indicate the case fatality rate.

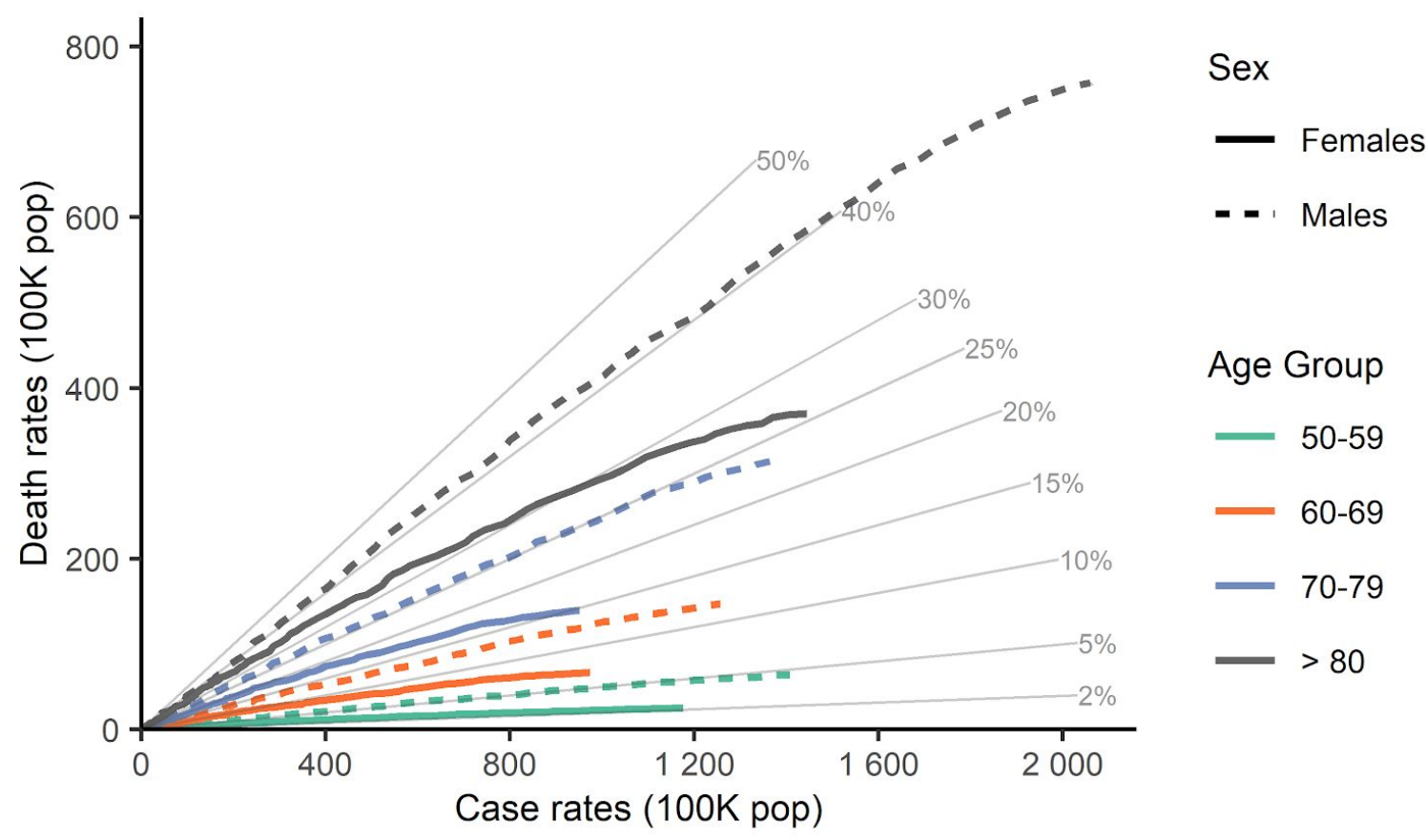

We repeat this exercise to compare Colombia with Mexico (see Fig. 3), where standardizing by population size is more justified. Case fatality rates and death rates are much higher in Mexico than in Colombia in each age band - around 2 -fold -, except for ages $80+$, which show a substantial reduction in the case fatality rate difference, and much higher death rates for Colombia. While we cannot separate the reasons for this with precision, it is clear that Colombia has conducted about twice as many tests per positive case than has Mexico, and positivity trends have been on the rise in both countries. ${ }^{8}$ However, we do not know the age pattern of positivity in these two countries. On the other hand, both countries have excellent open data practices, allowing for construction of detailed series taking into account retrospective corrections. This highlights challenges in making such comparisons, but also the need to produce data with sufficient detail to adjust for biases. It is our view that researchers should 
triangulate creatively from all available data rather than avoiding difficult comparisons.

Figure 3. Relation between deaths and cases per 100,000 population by age group in Mexico and Colombia, until 22 August 2020. Diagonal lines indicate the case fatality rate.

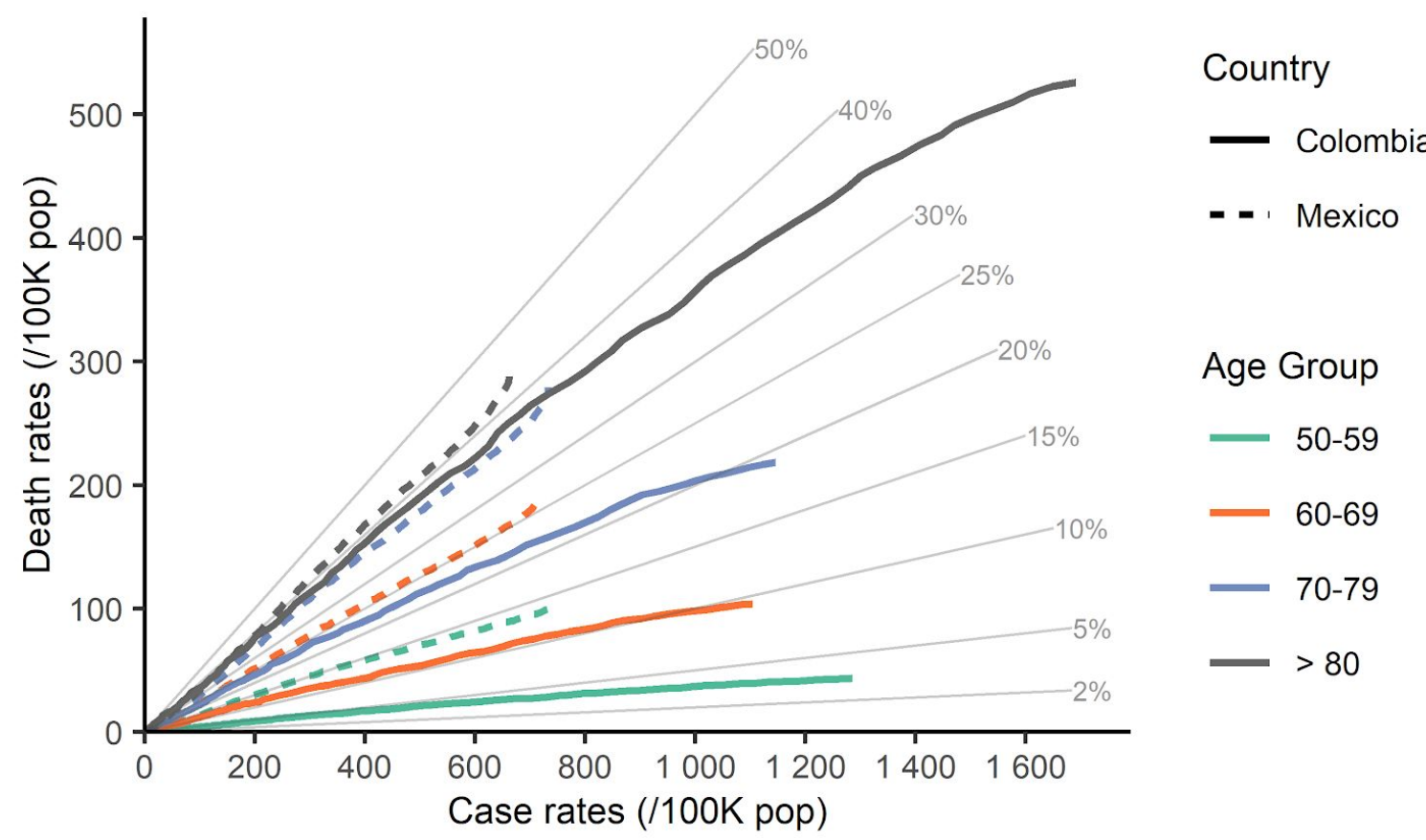

\section{Strengths and Weaknesses}

Since the beginning of the pandemic, it has been evident that population characteristics are key to understanding the prevalence, spread and fatality of COVID-19 across countries. However, data on cases, deaths, and tests disaggregated by age and sex are not easily comparable across countries, and sometimes not even accessible. The main strength of COVerAGE-DB is to provide a centralized, open-access, and fully reproducible repository of age-and sex-specific case, death, and test counts from COVID-19, collected from official sources, and harmonized to standard output formats. The data harmonization process is transparent, following a strict protocol. ${ }^{9}$ The initial input data is provided alongside the harmonized counts, as well as the code used to harmonize the different input measures, metrics, and age groups into comparable granular output metrics. All scripts are written in the open-source R programming language. ${ }^{10}$ The data sources and limitations are documented for each country in a standard metadata framework. 
A limitation of the COVerAGE-DB is the heterogeneous and difficult-to-evaluate quality of the underlying data. No single data source can currently claim accurate estimates of COVID-19 incidence or fatalities. Age-specific case counts are highly dependent upon the testing capacity, ${ }^{11}$ testing strategy, ${ }^{12}$ and differences in the definition of cases across sources and over time. Cases are underestimated everywhere, with underestimation expected to vary by age, given the relationship between age and case severity. ${ }^{13}$ The accuracy of diagnostic RT-PCR tests used to confirm infections is also known to vary. ${ }^{14}$ Furthermore, at any given date, cumulative counts are underestimated because of the lag between infection and a positive test result. ${ }^{15}$

Death counts from COVID-19 are also likely underestimated for similar reasons, but also due to various kinds of delays in death registration. Media reports have circulated about intentional data manipulation in some of the official data covered in the database. ${ }^{16}$ Excess all-cause mortality has been observed across many regions. ${ }^{17-20}$ Although some of these deaths are likely from postponing or foregoing treatment from non-COVID-19 related causes, the magnitude of this excess is suggestive that numerous COVID-19 related deaths are classified under different causes. Populations also differ in whether deaths to suspected COVID-19 cases are included in official statistics and in post-mortem practices when an infection is suspected. ${ }^{21}$ Some populations only report deaths occurring in hospitals, neglecting a potentially sizable proportion of deaths occurring in institutional settings and at home. ${ }^{22}$ While most populations currently report all deaths to confirmed COVID-19 infections as COVID-19 deaths for this database, the underlying cause of death eventually reported on death certificates may differ in patients with severe comorbidities. To mitigate biases and misinterpretations due to different practices and definitions, such information is constantly updated and documented in the metadata of the database, which is freely accessible to users.

All of these issues compromise the comparability of the data contained within the COVerAGE-DB, both across populations at any given time and within populations over time. For these reasons, infection fatality ratios, which include in the numerator detected and undetected COVID-19 infections, should not be estimated from COVerAGE-DB data alone. Proper estimation of incidence and fatality will likely require triangulating data across numerous sources as these become available. To this end, the COVerAGE-DB was designed to be easily merged with other databases such as the Our World In Data database on COVID-19 testing, ${ }^{8}$ the COVID-19 dashboard of Johns Hopkins, ${ }^{23}$ the World Population Prospects database, ${ }^{24}$ and the Short Term Mortality Fluctuations database.$^{20}$ Moreover, given that we have near-complete time series capturing the whole pandemic curve in some places, careful modeling of the lag structures might allow some of these data-driven biases to be estimated. 


\section{Data Resource Access}

Both merged input and harmonized output files can be downloaded directly from the OSF site (https://osf.io/mpwjq, DOI: 10.17605/OSF.IO/MPWJO), which contains a folder called Data with three files of primary data. Fig 4 shows where to find the files in the OSF repository.

Figure 4. View of the Open Science Framework (OSF) repository, File section (https://osf.io/mpwjq/files/). To download data files, click on Data, and select one of the files.

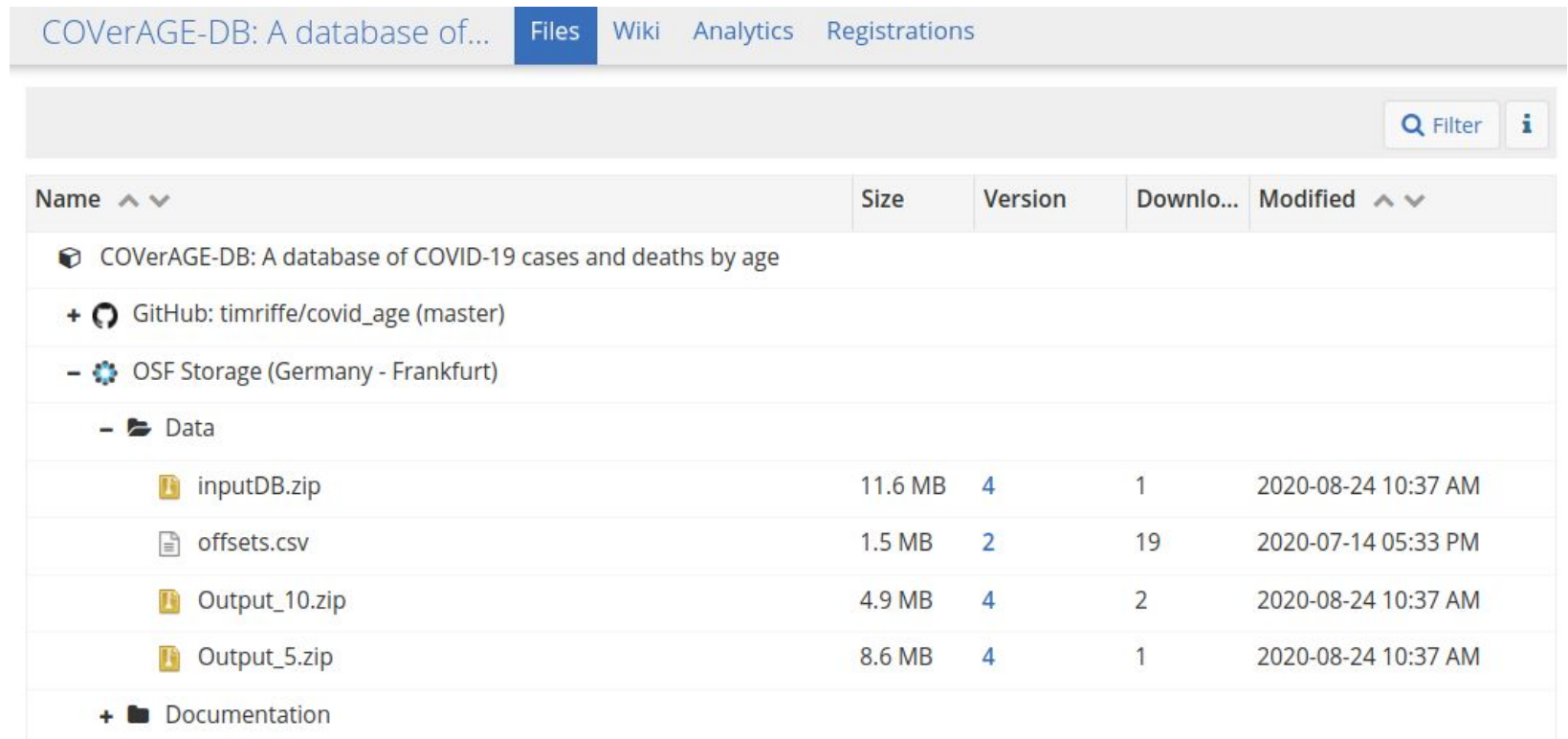

Each of the main data files has a stable link (see Tab. 1), which always points to the most recent version. Each file is a zipped csv file by the same name.

Table 1. The main data files, a description of their content, and their stable URLs.

Filename

Description

Stable URL

$\begin{array}{ll}\text { 1. inputDB.zip } & \text { Data in original metrics, } \\ \text { measures, and age intervals }\end{array}$

[https://osf.io/9dsfk/]

Data with standardized metrics

2.Output_5.zip and measures, and harmonized

[https://osf.io/7tnfh/ ] age groups in 5-year intervals

Data with standardized metrics

3. Output_10.zip and measures, and harmonized

[https://osf.io/43ucn] age groups in 10-year intervals 
For stable links to download particular versions, click on the version number in the Version column seen in Fig. 4. Users can note versions either by referring to timestamps provided in the headers of data files or by referring to OSF file version numbers, which increment with each daily update.

A data dictionary is given in both the OSF wiki

(https://osf.io/mpwjq/wiki/home/) and the Method Protocol. Files are shared in csv format to be as universally accessible as possible. A guide to getting started using the data in $\mathrm{R}$ is also provided (https://bit.ly/3g8nIVU), and tips for other statistical packages may also be added. Users are encouraged to reach out for further information or advice on using the database, or to express interest in the project: coverage-db@demogr.mpg.de.

\section{Acknowledgments}

We gratefully acknowledge the hard work of health ministries and statistical offices around the world in preparing and disseminating the data included in COVerAGE-DB.

\section{Notes}

The COVerAGE-DB project team is composed of more than 60 researchers that share in the authorship of this manuscript according to the CRediT authorship system (see contributions by author under the following link:

https://docs.google.com/spreadsheets/d/1SHygPJArbfInXlmFku6vR7LzLyG zoop1ZkBaPdanAIw/edit?usp=sharing).

\begin{tabular}{|l|l|}
\hline \multicolumn{1}{|c|}{ Last, First } & \multicolumn{1}{c|}{ Affiliation } \\
\hline Riffe, Tim & $\begin{array}{l}\text { Max Planck Institute for Demographic Research, } \\
\text { Germany }\end{array}$ \\
\hline Acosta, Enrique & $\begin{array}{l}\text { Max Planck Institute for Demographic Research, } \\
\text { Germany }\end{array}$ \\
\hline Aburto, José Manuel & University of Oxford, UK \\
\hline Alburez-Gutierrez, Diego & $\begin{array}{l}\text { Max Planck Institute for Demographic Research, } \\
\text { Germany }\end{array}$ \\
\hline Altová, Anna & Faculty of Science, Charles University, Czechia \\
\hline Basellini, Ugofilippo & $\begin{array}{l}\text { Max Planck Institute for Demographic Research, } \\
\text { Germany }\end{array}$ \\
\hline Bignami, Simona & University of Montreal, Canada \\
\hline Breton, Didier & Université de Strasbourg, France \\
\hline Choi, Eungang & Ohio State University, USA \\
\hline Cimentada, Jorge & $\begin{array}{l}\text { Max Planck Institute for Demographic Research, } \\
\text { Germany }\end{array}$ \\
\hline
\end{tabular}




\begin{tabular}{|c|c|}
\hline De Armas, Gonzalo & Universidad de la República, Uruguay \\
\hline Del Fava, Emanuele & $\begin{array}{l}\text { Max Planck Institute for Demographic Research, } \\
\text { Germany }\end{array}$ \\
\hline Delgado, Alicia & Pontificia Universidad Católica del Ecuador, Ecuador \\
\hline Diaconu, Viorela & $\begin{array}{l}\text { Max Planck Institute for Demographic Research, } \\
\text { Germany }\end{array}$ \\
\hline Donzowa, Jessica & $\begin{array}{l}\text { Max Planck Institute for Demographic Research, } \\
\text { Germany }\end{array}$ \\
\hline Dudel, Christian & $\begin{array}{l}\text { Max Planck Institute for Demographic Research, } \\
\text { Germany }\end{array}$ \\
\hline Fröhlich, Antonia & $\begin{array}{l}\text { Max Planck Institute for Demographic Research, } \\
\text { Germany }\end{array}$ \\
\hline Gagnon, Alain & University of Montreal, Canada \\
\hline Garcia Cristómo, Mariana & El Colegio de México, México \\
\hline Garcia-Guerrero, Victor M. & El Colegio de México, México \\
\hline González-Díaz, Armando & El Colegio de México, México \\
\hline Hecker, Irwin & Université de Strasbourg, France \\
\hline Koba, Dagnon Eric & L'Agence Française de Développement , France \\
\hline Kolobova, Marina & $\begin{array}{l}\text { Max Planck Institute for Demographic Research, } \\
\text { Germany }\end{array}$ \\
\hline Kühn, Mine & $\begin{array}{l}\text { Max Planck Institute for Demographic Research, } \\
\text { Germany }\end{array}$ \\
\hline Liu, Chia & St. Andrews University, UK \\
\hline Lozer, Andrea & $\begin{array}{l}\text { Max Planck Institute for Demographic Research, } \\
\text { Germany }\end{array}$ \\
\hline Manea, Mădălina & Research Institute for the Quality of Life, Romania \\
\hline Masum, Muntasir & Univ. Texas San Antonio, USA \\
\hline Mogi, Ryohei & Centre for Demographic Studies, Spain \\
\hline Morwinsky, Saskia & $\begin{array}{l}\text { Max Planck Institute for Demographic Research, } \\
\text { Germany }\end{array}$ \\
\hline Musizvingoza, Ronald & Bursa Uludag University, Turkey \\
\hline Myrskylä,Mikko & $\begin{array}{l}\text { Max Planck Institute for Demographic Research, } \\
\text { Germany }\end{array}$ \\
\hline Nepomuceno, Marília R. & $\begin{array}{l}\text { Max Planck Institute for Demographic Research, } \\
\text { Germany }\end{array}$ \\
\hline Nickel, Michelle & $\begin{array}{l}\text { Max Planck Institute for Demographic Research, } \\
\text { Germany }\end{array}$ \\
\hline Nitsche, Natalie & $\begin{array}{l}\text { Max Planck Institute for Demographic Research, } \\
\text { Germany }\end{array}$ \\
\hline Oksuzyan, Anna & $\begin{array}{l}\text { Max Planck Institute for Demographic Research, } \\
\text { Germany }\end{array}$ \\
\hline
\end{tabular}




\begin{tabular}{|c|c|}
\hline Oladele, Samuel & Federal University Oye Ekiti, Nigeria \\
\hline Olamijuwon, Emmanuel & University of the Witwatersrand, South Africa \\
\hline Omodara, Oluwafunke & Federal University Oye Ekiti, Nigeria \\
\hline Ouedraogo, Soumaila & French Institute for Demographic Studies, France \\
\hline Paredes, Mariana & Universidad de la República, Uruguay \\
\hline Pascariu, Marius & SCOR, France \\
\hline Piriz, Manuel & Universidad de la República, Uruguay \\
\hline Pollero, Raquel & Universidad de la República, Uruguay \\
\hline Rehermann, Federico & Universidad de la República, Uruguay \\
\hline Ribeiro, Filipe & CIDEHUS, Universidade de Évora, Portugal \\
\hline Rizzi, Silvia & University of Southern Denmark, Denmark \\
\hline Rowe, Francisco & University of Liverpool, UK \\
\hline Sasson, Isaac & Tel Aviv University, Israel \\
\hline Shi, Jiaxin & $\begin{array}{l}\text { Max Planck Institute for Demographic Research, } \\
\text { Germany }\end{array}$ \\
\hline Silva-Ramirez, Rafael & University of Montreal, Canada \\
\hline Strozza, Cosmo & University of Southern Denmark, Denmark \\
\hline Torres, Catalina & Muséum national d'histoire naturelle, France \\
\hline Trias-Llimos, Sergi & Centre for Demographic Studies, Spain \\
\hline Uchikoshi, Fumiya & Princeton University, USA \\
\hline van Raalte, Alyson & $\begin{array}{l}\text { Max Planck Institute for Demographic Research, } \\
\text { Germany }\end{array}$ \\
\hline Vazquez-Castillo, Paola & El Colegio de México, México \\
\hline Vilela, Estevão & Universidade Federal de Minas Gerais, Brazil \\
\hline Williams, Iván & Universidad de Buenos Aires / INDEC, Argentina \\
\hline Zarulli, Virginia & University of Southern Denmark, Denmark \\
\hline
\end{tabular}

\section{References}

1. Rizzi S, Gampe J, Eilers PHC. Efficient Estimation of Smooth Distributions From Coarsely Grouped Data. Am J Epidemiol. Oxford Academic; 2015 Jul 15;182(2):138-147.

2. Medford A, Trias-Llimós S. Population age structure only partially explains the large number of COVID-19 deaths at the oldest ages. Demographic Research. 2020 Aug 21;43(19):533-544.

3. Dudel C, Riffe T, Acosta E, Raalte AA van, Strozza C, Myrskyla M. Monitoring trends and differences in COVID-19 case fatality rates using decomposition methods:

Contributions of age structure and age-specific fatality. medRxiv. Cold Spring Harbor Laboratory Press; 2020 May 18;2020.03.31.20048397.

4. Bohk-Ewald C, Dudel C, Myrskyla M. A demographic scaling model for estimating the 
total number of COVID-19 infections. medRxiv. Cold Spring Harbor Laboratory Press; 2020 May 26;2020.04.23.20077719.

5. Arpino B, Bordone V, Pasqualini M. No clear association emerges between intergenerational relationships and COVID-19 fatality rates from macro-level analyses. PNAS. National Academy of Sciences; 2020 Aug 11;117(32):19116-19121.

6. Pifarré i Arolas H, Acosta E, Casasnovas GL, et al. Global years of life lost to COVID-19 [Internet]. SocArXiv; 2020 Jun. Available from: https://osf.io/gveaj

7. Kashnitsky I, Aburto JM. COVID-19 in unequally ageing European regions. World Development [Internet]. forthcoming [cited 2020 Aug 20]; Available from: https://osf.io/abx7s/

8. Roser M, Ritchie H, Ortiz-Ospina E, Hasell J. Coronavirus Pandemic (COVID-19). Our World in Data [Internet]. 2020 Mar 4 [cited 2020 Aug 17]; Available from: https://ourworldindata.org/coronavirus

9. Riffe T, Rizzi S, Dudel C, et al. Method Protocol for the COVerAGE-DB [Internet]. OSF; 2020 Apr p. 10. Report No.: 1. Available from: https://osf.io/jcnw3/

10. R Core Team. R: A Language and Environment for Statistical Computing [Internet]. Vienna, Austria: R Foundation for Statistical Computing; 2020. Available from: http://www.R-project.org

11. Cohen J, Kupferschmidt K. Countries test tactics in 'war' against COVID-19. Science. American Association for the Advancement of Science; 2020 Mar 20;367(6484):1287-1288.

12. Bi Q, Wu Y, Mei S, et al. Epidemiology and transmission of COVID-19 in 391 cases and 1286 of their close contacts in Shenzhen, China: a retrospective cohort study. The Lancet Infectious Diseases. Elsevier; 2020 Aug 1;20(8):911-919.

13. Verity R, Okell LC, Dorigatti I, et al. Estimates of the severity of coronavirus disease 2019: a model-based analysis. The Lancet Infectious Diseases [Internet]. Elsevier; 2020 Mar 30 [cited 2020 Apr 5];0(0). Available from: https://www.thelancet.com/journals/laninf/article/PIIS1473-3099(20)30243-7/abstract

14. Tang Y-W, Schmitz JE, Persing DH, Stratton CW. Laboratory Diagnosis of COVID-19: Current Issues and Challenges. Journal of Clinical Microbiology [Internet]. American Society for Microbiology Journals; 2020 May 26 [cited 2020 Aug 17];58(6). Available from: https://jcm.asm.org/content/58/6/e00512-20

15. Backer JA, Klinkenberg D, Wallinga J. Incubation period of 2019 novel coronavirus (2019-nCoV) infections among travellers from Wuhan, China, 20-28 January 2020. Eurosurveillance. European Centre for Disease Prevention and Control; 2020 Feb 6;25(5):2000062.

16. Leon DA, Shkolnikov VM, Smeeth L, Magnus P, Pechholdová M, Jarvis CI. COVID-19: a need for real-time monitoring of weekly excess deaths. The Lancet. Elsevier; 2020 May 2;395(10234):e81.

17. Wu J, McCann A, Katz J, Peltier E. 161,000 Missing Deaths: Tracking the True Toll of the Coronavirus Outbreak. The New York Times [Internet]. [cited 2020 Aug 17];

Available from: https://www.nytimes.com/interactive/2020/04/21/world/coronavirus-missing-deaths.html

18. The Economist. Tracking covid-19 excess deaths across countries. The Economist [Internet]. [cited 2020 Aug 17]; Available from: https://www.economist.com/graphic-detail/2020/07/15/tracking-covid-19-excess-deaths- 
across-countries

19. Mølbak K, Mazick A. European monitoring of excess mortality for public health action (EuroMOMO)Kåre Mølbak. Eur J Public Health [Internet]. Oxford Academic; 2013 Oct 1 [cited 2020 Aug 17];23(suppl_1). Available from: https://academic.oup.com/eurpub/article/23/suppl_1/ckt126.113/2837977

20. Human Mortality Database, University of California, Berkeley (USA), Max Planck Institute for Demographic Research (Germany). Short-term Mortality Fluctuations (STMF) data series [Internet]. The Human Mortality Database 2020 [cited 2020 May 28]. Available from: http://www.mortality.org/

21. INED. Demographics of COVID-19 Deaths [Internet]. Ined - Institut national d'études démographiques 2020 [cited 2020 Aug 17]. Available from: https://dc-covid.site.ined.fr/en/

22. Meslé F, Pison G. Comment la France compte-t-elle ses morts ? [Internet]. The Conversation [cited 2020 Aug 17]. Available from: http://theconversation.com/comment-la-france-compte-t-elle-ses-morts-135586

23. Dong E, Du H, Gardner L. An interactive web-based dashboard to track COVID-19 in real time. The Lancet Infectious Diseases. Elsevier; 2020 May 1;20(5):533-534.

24. World Population Prospects - Population Division - United Nations [Internet]. [cited 2020 Aug 17]. Available from: https://population.un.org/wpp/ 